\title{
Swimming improves high-fat induced insulin resistance by regulating lipid and energy metabolism and the insulin pathway in rats
}

\author{
AN SONG ${ }^{1}, \mathrm{CHAO} \mathrm{WANG}^{2}$, LUPING REN ${ }^{2}$ and JIAJUN ZHAO ${ }^{1}$ \\ ${ }^{1}$ Department of Endocrinology, Shandong Provincial Hospital, Shandong University, Jinan, Shandong 250021; \\ ${ }^{2}$ Department of Endocrinology, General Hospital of Hebei, Shijiazhuang, Hebei 050051, P.R. China
}

Received January 12, 2014; Accepted April 2, 2014

DOI: $10.3892 / \mathrm{ijmm} \_x x x x x x x x$

\begin{abstract}
In this study, we aimed to determine the preventive and therapeutic effects of swimming on insulin resistance in high-fat-fed rats. Sprague-Dawley rats were divided into 4 groups and fed for 8 weeks as follows: i) the control (Con) group fed a control diet; ii) the high-fat (HF) group fed a high-fat diet; iii) the treatment (ST) group fed a high-fat diet and trained with swimming from the 4th week; and iv) the prevention (SP) group fed a high-fat diet and trained with swimming from the 1st week of the experiment. A hyperinsulinemic-euglycemic clamp was used to evaluate the insulin sensitivity of the rats. The ultrastructure of the liver cells was observed by electron microscopy. Hepatic lipid accumulation was observed by Oil Red O staining. Quantitative RT-PCR and western blot analysis were performed to detect the expression of proteins related to lipid metabolism, energy metabolism and insulin signaling transduction. After 8 weeks of feeding, compared with the Con group, the glucose infusion rate (GIR) was significantly decreased; a significant lipid accumulation was observed in the liver, while the ultrastructure of the liver cells was damaged in the HF group. Proteins related to lipid metabolism in the liver and skeletal muscle, including FAT and FABP were upregulated, while CPT1 and PPAR levels were downregulated in the HF group. The levels of the energymetabolism-related molecules, AMPK $\alpha 2$, PGC1 $\alpha$, PGC1 $\beta$ and MFN2 were downregulated in skeletal muscle in the HF group. The expression levels of insulin signaling transduction molecules, INSR, IRS1, PI3K/p85, AKT2 and GLUT4, as well as the phosphorylation levels of INSR, IRS1, PI3K/p85 and AKT2 were lower in skeletal muscles in the HF rats. Compared with HF group, the GIR levels were significantly increased in
\end{abstract}

Correspondence to: Professor Jiajun Zhao, Department of Endocrinology, Shandong Provincial Hospital, Shandong University, 324 Jingwuweiqi Road, Jinan, Shandong 250021, P.R. China

Email: zhaojiajunsa@163.com

Key words: swimming, high-fat diet, insulin resistance, metabolism the ST and SP groups. Lipid accumulation and damage to the ultrastructure of the liver cells were improved in both groups. The expression of molecules related to lipid metabolism in the liver and skeletal muscle, energy metabolism in skeletal muscle and insulin signaling transduction were all markedly upregulated. In conclusion, swimming can effectively improve insulin sensitivity and even prevent insulin resistance by affecting the expression of proteins related to lipid metabolism, energy metabolism and insulin signaling transduction in rats fed a high-fat diet.

\section{Introduction}

Metabolic diseases, such as diabetes, hypertension, high cholesterol levels and coronary heart disease, have become increasingly prevalent $(1,2)$. Therefore, finding a reasonable method of reducing the risk of developing metabolic disease has become a global concern. Insulin resistance, defined as the diminished ability of insulin-sensitive tissues to respond to insulin, plays a central role in the development of metabolic diseases. Insulin resistance is caused by the decreased ability of peripheral target tissues, including liver, muscle and fat, to respond properly to normal circulating concentrations of insulin $(3,4)$. Studies have shown that the prolonged lack of exercise and a high-fat diet are the main causes of insulin resistance, whereas regular exercise, moderate weight loss, as well as a low-fat and low-calorie diet can improve insulin sensitivity (5-7).

High energy intake from a high-fat diet may result in fat deposition in the liver and skeletal muscle (8). A large number of epidemiological data have demonstrated that the incidence of insulin resistance and type 2 diabetes in individuals consuming a high-fat diet for a prolonged period of time is significantly higher than that in the 'normal' population $(9,10)$. Animal experiments have also confirmed that fat deposition can damage the insulin-sensitive organs, such as skeletal muscle and the liver (11). Recent studies have suggested that lipid accumulation in skeletal muscle and the liver correlates closely with insulin resistance $(12,13)$.

The liver and skeletal muscles are the main organs of the body where glucose and lipid uptake and utilization occur; they are also the most important sites for energy metabolism 
based on the role of insulin. Increased fatty acid intake and/or decreased lipid oxidative capacity are the main causes of lipid accumulation in the liver and skeletal muscle (14-16).

Exercise can rapidly increase energy consumption, promote aerobic oxidation in vivo, and increase the activity of lipoprotein lipase and $\beta$ oxidation of fatty acid, thus reducing fat deposition (16). Decreased fat accumulation in the liver and skeletal muscle may increase the insulin receptor (INSR) binding activity to insulin on the cell membrane, and improve insulin resistance (15). Swimming is a popular sport, and may be beneficial in the prevention and treatment of metabolic diseases.

In the present study, a rat model of insulin resistance was established by feeding rats a high-fat diet, and the effects of swimming on fat deposition and insulin resistance were observed. The ultrastructural changes in liver tissue were also observed. Furthermore, fat metabolism, energy metabolism and the expression of genes related to the insulin signaling pathway were detected in the liver and muscle tissue in order to explore the effects of swimming on high-fat diet-induced insulin resistance.

\section{Materials and methods}

Animals and groups. Male Sprague-Dawley (SD) rats weighing approximately 200-220 g were housed in wire-bottom cages to prevent coprophagia. The environment was controlled in terms of light (12:12-h light-dark cycle commencing at 6:00 a.m.), humidity and room temperature $\left(20-23^{\circ} \mathrm{C}\right)$. Apart from pretest overnight fasting and the immediate post-operative period, the animals were allowed free access to water and chow. The normal rodent chow diet was obtained from the Hebei Medical University Animal Laboratory (Hebei, China) and contained $10.3 \%$ fat, $24.2 \%$ protein and $65.5 \%$ carbohydrate (kcal). The high-fat diet consisted of $59.8 \%$ fat, $20.1 \%$ protein and $20.1 \%$ carbohydrate $(\mathrm{kcal})$. The animal experiments were conducted according to protocols reviewed and approved by the Animal Experimental Ethics Committee of Hebei General Hospital, Shijiazhuang, China (approval ID: HBGH-2012005).

Seven days after their arrival, the rats were randomly divided into 4 groups ( $n=10$ per group): i) the normal control group (Con) group fed the control (normal) diet for 8 weeks, ii) the high-fat (HF) group fed the high-fat-diet for 8 weeks, iii) the swimming treatment (ST) group fed the high-fat-diet for 8 weeks and trained with swimming sports from the end of the 4th week and iv) the swimming prevention (SP) group fed the high-fat-diet and trained with swimming exercise from the beginning of the epxeriment. Body weight, food and water intake, fasting glucose, insulin lipids and insulin sensitivity were measured at the end of the 4th and the 8th week; the rats were then sacrificed, and the liver and skeleton muscle tissue samples were taken immediately and kept at $-80^{\circ} \mathrm{C}$ after being quick-frozen in liquid nitrogen.

Swimming. Swimming was performed in a $100 \times 50 \times 50 \mathrm{~cm}$ aquarium, containing clean tap water to a depth of approximately $2 / 3$. Water temperature was maintained at $30 \pm 2^{\circ} \mathrm{C}$. The animals in the Con group were placed into the water, but were taken out immediately without any swimming exercise. The animals in the other groups swam for 10 min daily in the initial 2 days, and then their swimming time was gradually increased to 45 min daily. All rats swam 5 days/week (2 days of rest). Rats in the SP group swam for 8 weeks, while the ST rats swam from the 5 th to the 8 th week.

Biochemistry assay. Blood samples were obtained from the abdominal aorta. Blood glucose (BG) levels were measured using an Accu-chek Active Meter (ACCU-CHEK ${ }^{\circledR}$ Active; Roche Diagnostics GmbH, Mannheim, Germany) and insulin levels were analyzed using a Rat Insulin ELISA kit (Crystal Chem Inc., Downers Grove, IL, USA). Total cholesterol (TC) and triglyceride (TG) levels were measured on an automatic biochemistry analyzer (Beckman X20; Beckman Coulter, Brea, CA, USA).

Lee index. The body weight and length of the rats from nose to anus were measured on the 4th and 8th week, and the Lee index was calculated using the following formula: $[3 \sqrt{ }$ weight $g) /$ length $(\mathrm{cm})] \times 1,000$.

Oil Red $O$ staining. Frozen sections of liver tissues were used for Oil Red O staining of lipid deposits. The selected specimens were thin-sectioned, viewed and photographed under an electron microscope (Hitachi H7500; Hitachi Ltd., Tokyo, Japan).

Hyperinsulinemic euglycemic clamp. The insulin sensitivity of the rats was measured using a hyperinsulinemic euglycemic clamp as previously described (11). The rats were put under general anesthesia (3\% pelltobarbitalum natricum; $60 \mathrm{mg} /$ $\mathrm{kg}$, intraperitoneally), then catheters were inserted into the right jugular vein and carotid arteries of the rats; the catheters were subcutaneously exteriorized from the back of the neck. The catheters were flushed with isotonic saline containing heparin $(50 \mathrm{U} / \mathrm{ml})$. The rats were allowed to recover for 3 days. Hyperinsulinemic euglycemic clamps were used on fasted, awake and unrestrained animals. Insulin $(4 \mathrm{mU} / \mathrm{kg} / \mathrm{min})$ was infused through the jugular vein catheter for 0 to $90 \mathrm{~min}$. A variable rate infusion of $30 \%$ glucose was used. BG levels were monitored using a glucometer (ACCU-CHEK ${ }^{\circledR}$ Active; Roche Diagnostics $\mathrm{GmbH}$ ) and the glucose infusion rate (GIR) was adjusted every $5 \mathrm{~min}$ as required. Stable GIRs were obtained within approximately $60 \mathrm{~min}$ of the insulin infusion and maintained thereafter.

Electron microscopy. Tissue samples were cut into small sections $(1 \times 1 \times 2 \mathrm{~mm})$ and fixed in $2.5 \%$ glutaraldehyde, post-fixed in $1 \%$ osmium tetroxide, dehydrated and then the orientated longitudinal sections were embedded in Epon. The initial low-power screening of semi-thin $(300 \mathrm{~nm})$ sections stained with Toluidine blue was then carried out to optimize the plane of sectioning, then ultra-thin $(60 \mathrm{~nm})$ longitudinal sections of each sample were cut. The sections were mounted on copper grids and then stained with lead citrate and uranyl acetate. Each sample was examined using a transmission electron microscope (TEM; Hitachi H-7500; Hitachi Ltd.) at an accelerating voltage of $80 \mathrm{kV}$.

Quantitative RT-PCR. Total RNA of all samples was extracted using a standard TRIzol (Invitrogen Life Technologies, 
Table I. Primer sequences for PCR.

\begin{tabular}{lrlll}
\hline Gene & $\begin{array}{r}\text { Size } \\
(\mathrm{b})\end{array}$ & \multicolumn{1}{c}{ Forward $\left(5^{\prime} \rightarrow 3^{\prime}\right)$} & & \multicolumn{1}{c}{ Reverse (5' $\left.\rightarrow 3^{\prime}\right)$} \\
GLUT4 & 62 & CCCACAAGGCACCCTCACTA & TGCCACCCACAGAGAAGATG & NenBank no. \\
AMPK $\alpha 2$ & 133 & ACAGAAGCCAAATCAGGGACT & CACGGATGAGGTAAGAGAGACT & NM_019142 \\
PPAR $\alpha$ & 71 & GGACTTGAATGACCAGGTTACC & AGGAGGACAGCATCGTGAAG & NM_013196 \\
PPAR $\gamma$ & 68 & ACCAGGGAGTTCCTCAAAAGC & GCAAACTCAAACTTAGGCTCCATAA & NM_013124 \\
CPT1B & 87 & CCAGGCAAAGAGACAGACTTG & GCCAAACCTTGAAGAAGCGA & NM_013200 \\
CPT1A & 132 & TTATCGTGGTGGTGGGTG & CGCTCACAATGTTCTTCGTCT & NM_031559 \\
PGC1 $\alpha$ & 168 & AGCCACTACAGACACCGCAC & CCTTTCAGACTCCCGCTTC & NM_031347 \\
PGC1 $\beta$ & 72 & GATTCCGAGTTCTTCCAGATTG & GTCATCCAGGGTCTTGGTAAG & NM_176075 \\
MFN2 & 160 & AGCGTCCTCTCCCTCTGACA & TTCCACACCACTCCTCCGAC & NM_130894 \\
INSR & 135 & TTTGCCCAACCATCTGTAAG & GACCATCCAGGTAGAAGTTTCG & NM_017071 \\
IRS1 & 95 & AAGGAGGTCTGGCAGGTTATC & ATGGTCTTGCTGGTCAGGC & NM_012969 \\
PI3K/p85 & 135 & GCCTGCTCTGTAGTGGTAGATG & GGAGGTGTGTGGTAATGTAGC & NM_013005 \\
AKT2 & 79 & CTGAGATGATGGAGGTAGCG & CCGAGGAGTTTGAGATAATCG & NM_017093 \\
FAT & 71 & GTTCAGAAACCAAGTGACCG & CTCCAACACCAAGTAAGACCAT & NM_031561 \\
FABP1 & 78 & TCAAAGGCATAAAGTCCGTG & TGTAGACGATGTCACCCAGTG & NM_012556 \\
FABP3 & 91 & AAGGTCAAGTCGGTCGTGA & TTAGTTCCCGTGTAAGCGTAGT & NM_024162 \\
GAPDH & 120 & TGAACGGGAAGCTCACTGG & GCTTCACCACCTTCTTGATGTC & NM_017008 \\
\hline
\end{tabular}

Carlsbad, CA, USA) RNA isolation method. Reverse transcription was carried out according to the instructions of the Easy Script First-Strand cDNA Synthesis Super Mix kit (TransGen Biotech Co., Ltd., Beijing, China). Quantitative PCR was performed on an ABI-7300 PCR System (Applied Biosystems, Foster City, CA, USA) using the SYBR-Green I GoTaq ${ }^{\circledR}$ qPCR Master Mix (Promega, Madison, WI, USA) kit. PCR was performed as follows: 1 cycle at $95^{\circ} \mathrm{C}$ for $5 \mathrm{~min}$, followed by 40 cycles at $95^{\circ} \mathrm{C}$ for $15 \mathrm{sec}, 58^{\circ} \mathrm{C}$ for $20 \mathrm{sec}$ and $72^{\circ} \mathrm{C}$ for 30 sec. The primer sequences for PCR are presented in Table I. The gene expression of each sample was analyzed in duplicate and normalized against the internal control gene, GAPDH. The results were expressed as relative gene expression as $2^{-\Delta \Delta \mathrm{Ct}}$ values. The relative expression of the target genes was obtained using the software SDS v1.3.2 provided with the PCR machine.

Western blot analysis. Western blot analysis was performed as previously described (17). In brief, the tissues were treated with lysis buffer [1\% Triton X-100, $150 \mathrm{mM} \mathrm{NaCl}, 10 \mathrm{mM}$ Tris- $\mathrm{HCl}$ (pH 7.4); 1 mM EDTA, 1 mM EGTA (pH 8.0); 0.2 mM phenylmethylsulfonyl fluoride, $0.2 \mathrm{mM} \mathrm{Na}_{3} \mathrm{VO}_{4}$ and $0.5 \%$ NP-40]. Equal amounts of protein from each sample were separated by $10 \%$ SDS-PAGE and electrotransferred onto PVDF membranes (Millipore, Bedford, MA, USA). The PVDF membranes were blocked with $5 \%$ BSA for $2 \mathrm{~h}$ at room temperature, and were subsequently incubated with the appropriate diluted primary antibodies to glucose transporter (GLUT)4, AMP-activated protein kinase (AMPK) $\alpha 2$, peroxisome proliferator-activated receptor (PPAR) $\alpha$, PPAR $\gamma$, carnitine palmitoyltransferase (CPT)1B, CPT1A, peroxisome proliferator activated receptor $\gamma$ co-activator (PGC) $1 \alpha$, PGC1 $\beta$, mitofusin (MFN)2, INSR, insulin receptor substrates (IRS)1, fatty acid translocase (FAT), fatty acid binding protein (FABP)1, FABP3, $\beta$-actin (all from Santa Cruz Biotechnology, Inc., Santa Cruz, CA, USA), phos- phatidylinositol-3-kinase (PI3K), p-PI3K/p85, protein kinase B (AKT) or p-AKT2 (all from Cell Signaling Technology, Inc., Billerica, MA, USA) overnight at $4^{\circ} \mathrm{C}$.. All the membranes were then incubated with the relevant secondary antibodies for $2 \mathrm{~h}$ at room temperature. Bands were detected with the enhanced chemiluminescence (ECL) detection system. $\beta$-actin served as an internal control protein.

Statistical analysis. Data are represented as the means \pm SD. Statistical analyses were performed using the SPSS statistical package (SPSS 13.0 software). One-way ANOVA was used to determine statistically significant differences between all groups. A value of $\mathrm{P}<0.05$ was considered to indicate a statistically significant difference.

\section{Results}

General changes in rats at the 4th week. As shown in Table II, at the 4 th week, no obvious changes were observed in the body weight, the TC levels or the Lee index of the rats in any of the groups $(\mathrm{P}>0.05)$. However, the fasting blood glucose (FBG), fasting serum insulin (FINS) and TG levels of the HF rats increased, while the GIR markedly decreased in the HF rats compared with the Con group $(\mathrm{P}<0.05)$. Furthermore, the FINS and TG levels of the rats in the SP group rats markedly decreased compared with those of the rats in the HF group $(\mathrm{P}<0.05)$. In particular, the TG levels in muscle tissue markedly increased in the HF rats compared with the Con group, but markedly decreased in the ST and SP group rats $(\mathrm{P}<0.05)$.

General changes in rats at the 8th week. As shown in Table III, at the $8^{\text {th }}$ week, no obvious changes were observed in the TC levels in any of the groups $(\mathrm{P}>0.05)$. However, the BG, FBG, FINS, TG and muscle TG levels, as well as the Lee index 
Table II. General changes in rats at the end of the 4th week.

\begin{tabular}{lrrrr}
\hline Factor & \multicolumn{1}{c}{ Con } & HF & ST & SP \\
\hline BW $(\mathrm{g})$ & $264.3 \pm 7.2$ & $276.4 \pm 10.5$ & $276.4 \pm 10.5$ & $267.1 \pm 6.4$ \\
FBG $\left(\mathrm{mmol} / \mathrm{l}^{-1}\right)$ & $3.51 \pm 0.25$ & $3.93 \pm 0.27^{\mathrm{a}}$ & $3.93 \pm 0.27$ & $3.64 \pm 0.27$ \\
FINS $\left(\mathrm{mU} / \mathrm{l}^{-1}\right)$ & $21.66 \pm 2.86$ & $26.17 \pm 2.56^{\mathrm{a}}$ & $26.17 \pm 2.56$ & $22.78 \pm 2.53^{\mathrm{b}}$ \\
Blood TG $\left(\mathrm{mmol} / \mathrm{l}^{-1}\right)$ & $0.72 \pm 0.14$ & $1.03 \pm 0.22^{\mathrm{a}}$ & $1.03 \pm 0.22$ & $0.75 \pm 0.24^{\mathrm{b}}$ \\
Muscle TG $(\mu \mathrm{mol} / \mathrm{g})$ & $1.87 \pm 0.22$ & $4.68 \pm 0.67^{\mathrm{a}}$ & $2.94 \pm 0.24^{\mathrm{b}}$ & $2.64 \pm 0.44^{\mathrm{b}}$ \\
TC $\left(\mathrm{mmol} / \mathrm{l}^{-1}\right)$ & $0.86 \pm 0.04$ & $1.04 \pm 0.17$ & $1.04 \pm 0.17$ & $0.91 \pm 0.16$ \\
Lee index & $301.18 \pm 5.52$ & $311.20 \pm 4.69$ & $311.20 \pm 4.69$ & $303.40 \pm 4.56$ \\
GIR $\left(\mathrm{mg} / \mathrm{kg}^{-1} \cdot \mathrm{min}^{-1}\right)$ & $11.42 \pm 1.29$ & $9.15 \pm 1.24^{\mathrm{a}}$ & $9.15 \pm 1.24$ & $10.93 \pm 1.33$ \\
\hline
\end{tabular}

${ }^{\mathrm{a}} \mathrm{P}<0.05$ vs. Con group; ${ }^{\mathrm{b}} \mathrm{P}<0.05$ vs. HF group. Con, control group; HF, group fed high-fat diet; $\mathrm{ST}$, treatment group fed high-fat diet and trained with swimming from week 4; SP, prevention group fed high-fat diet and trained with swimming from the start of the experiment; BW, body weight; FBG, fasting blood glucose; TG, triglyceride; TC, total cholesterol; GIR, glucose infusion rate.

Table III. General changes in rats at the end of the 8th week.

\begin{tabular}{lrrrr}
\hline Factor & \multicolumn{1}{c}{ Con } & HF & ST & SP \\
\hline BW $(\mathrm{g})$ & $285.4 \pm 8.5$ & $305.8 \pm 11.7^{\mathrm{a}}$ & $292.6 \pm 9.8$ & $289.3 \pm 7.9^{\mathrm{b}}$ \\
FBG $\left(\mathrm{mmol} / \mathrm{l}^{-1}\right)$ & $3.62 \pm 0.26$ & $4.52 \pm 0.24^{\mathrm{a}}$ & $4.04 \pm 0.32$ & $3.71 \pm 0.22^{\mathrm{b}}$ \\
FINS $\left(\mathrm{mU} / \mathrm{l}^{-1}\right)$ & $22.27 \pm 2.41$ & $32.33 \pm 4.98^{\mathrm{a}}$ & $25.57 \pm 4.19$ & $23.90 \pm 2.92^{\mathrm{b}}$ \\
Blood TG $\left(\mathrm{mmol} / 1^{-1}\right)$ & $0.76 \pm 0.10$ & $1.16 \pm 0.22^{\mathrm{a}}$ & $0.93 \pm 0.11$ & $0.81 \pm 0.20^{\mathrm{b}}$ \\
Muscle TG $(\mu \mathrm{mol} / \mathrm{g})$ & $2.30 \pm 0.28$ & $6.50 \pm 0.94^{\mathrm{a}}$ & $4.18 \pm 0.63^{\mathrm{b}}$ & $3.61 \pm 0.44^{\mathrm{b}}$ \\
TC $\left(\mathrm{mmol} / \mathrm{l}^{-1}\right)$ & $0.87 \pm 0.09$ & $1.10 \pm 0.19$ & $0.95 \pm 0.13$ & $0.95 \pm 0.13$ \\
Lee Index & $304.67 \pm 5.04$ & $320.71 \pm 5.05^{\mathrm{a}}$ & $309.48 \pm 5.33$ & $306.02 \pm 8.19^{\mathrm{b}}$ \\
GIR $\left(\mathrm{mg} / \mathrm{kg}^{-1} \cdot \mathrm{min}^{-1}\right)$ & $10.95 \pm 1.73$ & $8.19 \pm 1.77^{\mathrm{a}}$ & $9.43 \pm 1.51$ & $10.32 \pm 1.58^{\mathrm{b}}$ \\
\hline
\end{tabular}

${ }^{\mathrm{a}} \mathrm{P}<0.05$ vs. Con; ${ }^{\mathrm{b}} \mathrm{P}<0.05$ vs. HF. Con, control group; HF, group fed high-fat diet; ST, treatment group fed high-fat diet and trained with swimming from week 4; SP, prevention group fed high-fat diet and trained with swimming from the start of the experiment; BW, body weight; FBG, fasting blood glucose; TG, triglyceride; TC, total cholesterol; GIR, glucose infusion rate.

grade of the HF rats markedly increased compared with the rats in the Con group $(\mathrm{P}<0.05)$. Moreover, all of these indicators decreased in the SP group rats in comparison with the HF group rats $(\mathrm{P}<0.05)$. Most importantly, the GIR in the HF group rats markedly decreased when compared with that in the rats from the Con group $(\mathrm{P}<0.05)$; however, it returned to normal in the SP group $(\mathrm{P}<0.05)$. Nevertheless, there were no significant differences between the ST and the HF group as regards the above-mentioned indicators $(\mathrm{P}>0.05)$.

Swimming reduces lipid accumulation in the liver and improves ultrastructural damage to liver cells. The results of Oil Red O staining of the liver tissue indicated that there was a significant lipid deposition in the HF group rats; by contrast, in the ST and SP groups, lipid deposition was markedly reduced (Fig. 1).

As can be seen in Fig. 2, a substantial amount of lipid droplets were deposited in the HF group liver cells, the number of mitochondria and other organelles was markedly reduced, and the ultrastructure of the cells was extensively damaged. Although the amount of lipid droplets was reduced in the
SP and ST groups, the number of mitochondria and other organelles markedly increased and ultrastructural damage to the cells was markedly decreased in comparison with the HF group.

Swimming regulates fat metabolism-related gene expression in liver and skeletal muscle. In order to elucidate the effects of a high-fat diet and swimming on fat metabolism-related gene expression, the expression of 4 factors, FAT, CPT1, FABP and PPAR, was detected by quantitative RT-PCR and western blot analysis in the liver and skeletal muscle of the rats. The results revealed that the mRNA and protein expression of FAT and FABP1 was upregulated, while PPAR $\gamma$ and CPT1 $\alpha$ expression was downregulated in the liver of HF rats compared with the Con group. Following swimming, the expressoin of these 4 factors was upregulated in the ST and SP groups compared with the HF group (Fig. 3).

Similar results were observed in the skeletal muscle of rats. As shown in Fig. 4, FAT and FABP3 expression was upregulated, while PPAR $\alpha$ and CPT $1 \beta$ expression was downregulated in the skeletal muscle of rats in the HF group in comparison 

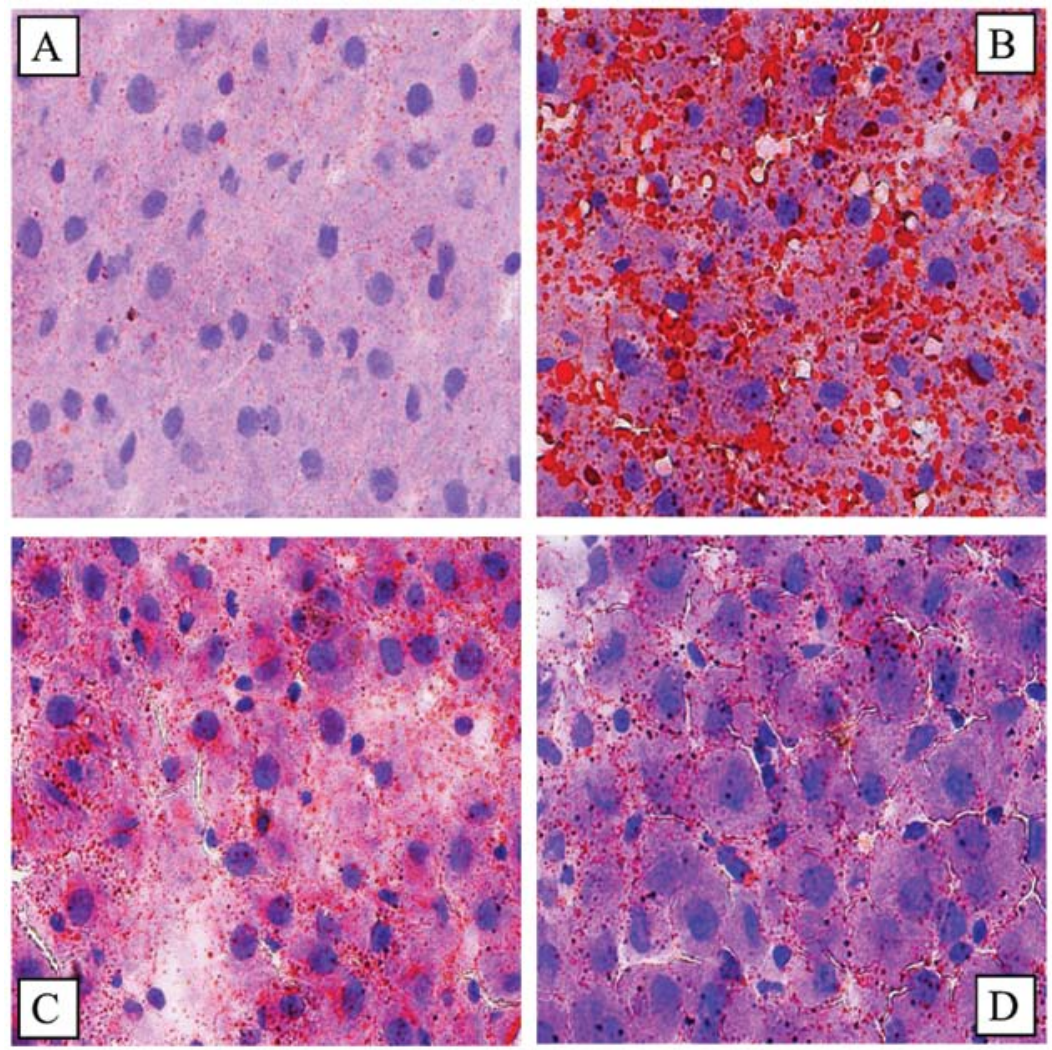

Figure 1. Swimming reduces lipid accumulation in the livers of rats. Rats were fed a control or high-fat diet for 8 weeks, and some were trained with swimming for 4 or 8 weeks; the rats were then sacrificed and liver tissues were obtained for Oil Red O staining to observe fat deposition in the livers of rats. Lipid deposition was stained red in the liver tissue. (A) Control group, (B) group fed high-fat diet (HF), (C) treatment group (ST) trained with swimming from week 4, (D) prevention group (SP) trained with swimming from the start of the experiment.
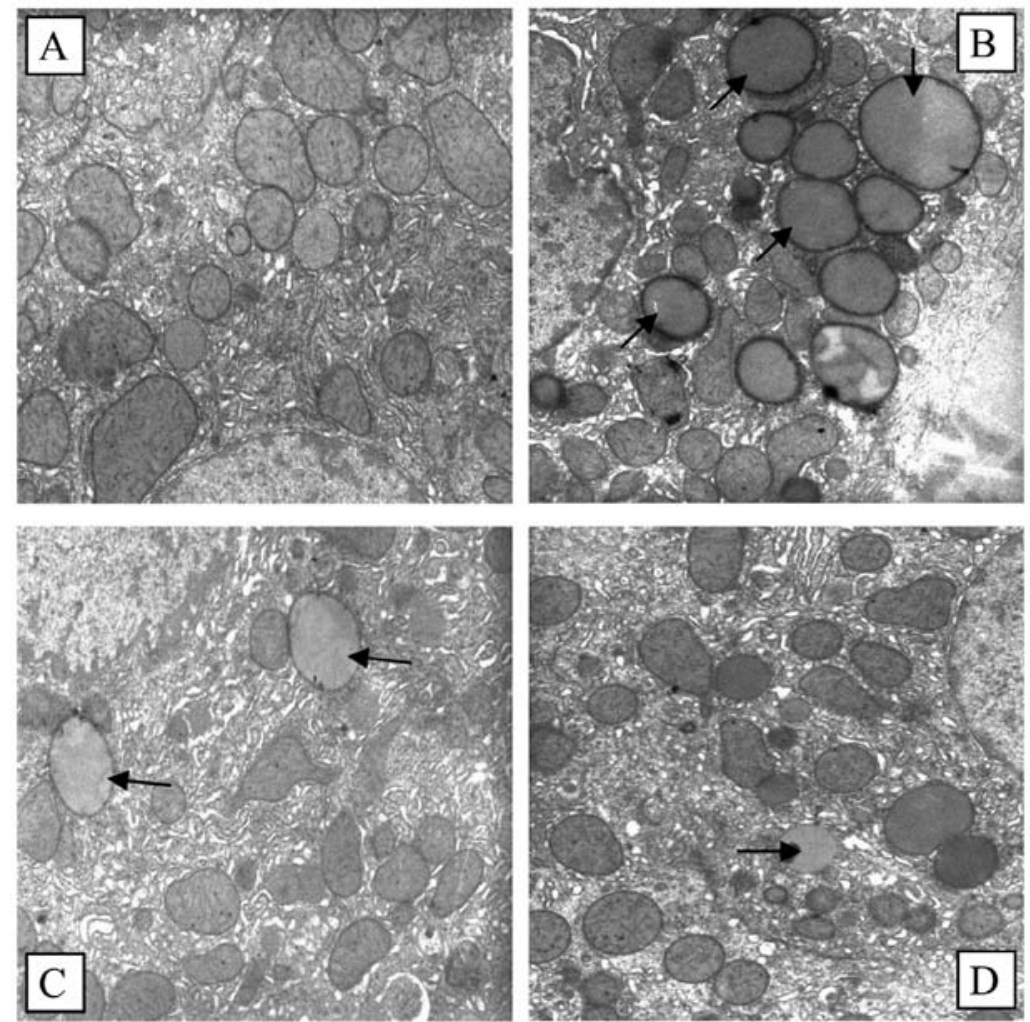

Figure 2. Swimming improves ultrastructure damage to liver cells. Rats were fed a control or high-fat diet for 8 weeks, and some were trained with swimming for 4 or 8 weeks; the rats were sacrificed. Liver tissues of rats were removed immediately for electron microscopy to indicate the ultrastructural changes in the liver cells. Lipid droplets in cells are marked by arrows. (A) Control group, (B) group fed high-fat diet (HF), (C) treatment group (ST) trained with swimming from week 4, (D) prevention group (SP) trained with swimming from the start of the experiment. 


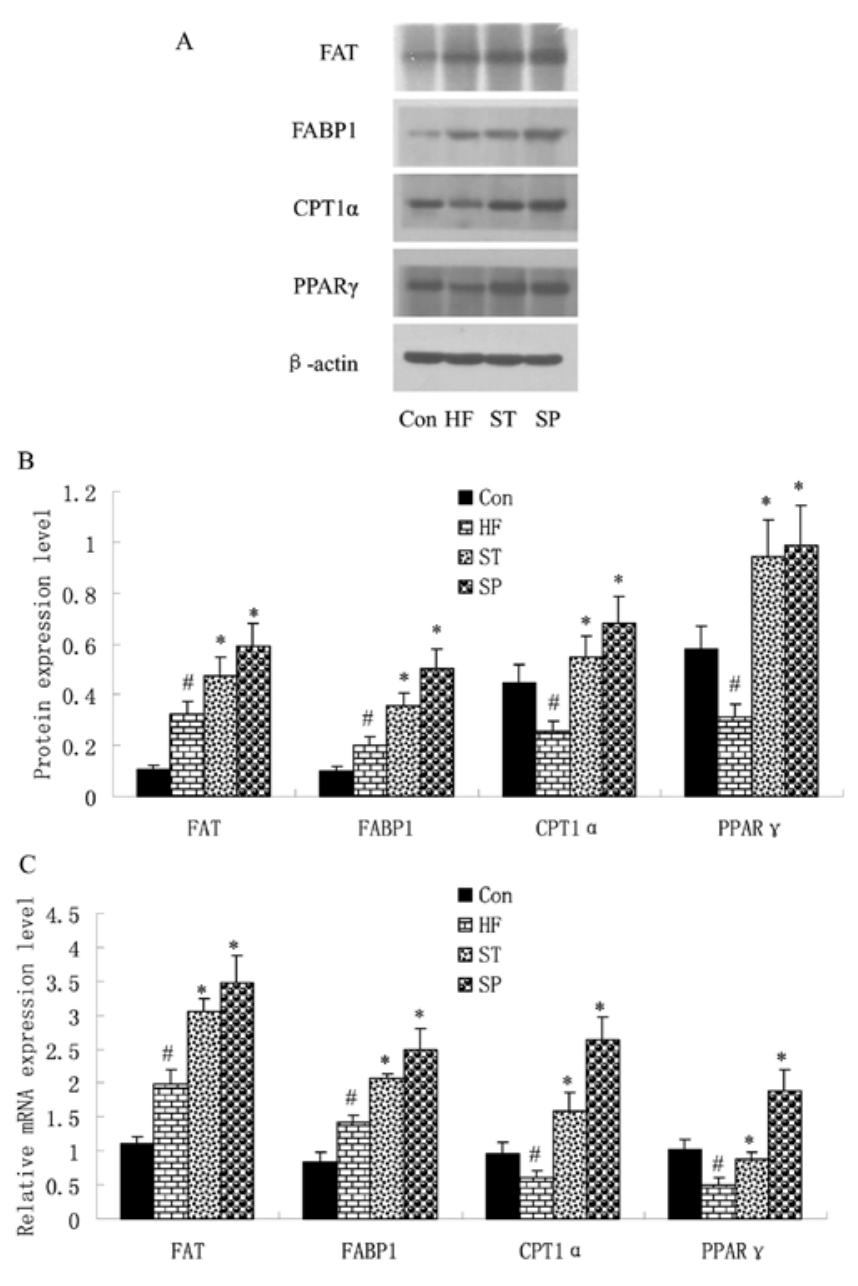

Figure 3. Swimming regulates fat metabolism-related gene expression in liver. Rats were fed a control or high-fat diet for 8 weeks, and trained with swimming exercise for 4 or 8 weeks, and were then sacrificed. Liver tissues of rats were removed immediately to determine the mRNA and protein expression levels of fat metabolism-related genes by (A) western blot analysis and (C) quantitative RT-PCR. The band density of western blots representing the target gene protein expression level is shown in (B). Experiments were repeated 3 times $(n=3) .{ }^{~} \mathrm{P}<0.05$ vs. Con group; ${ }^{*} \mathrm{P}<0.05$ vs. HF group. Con, control group; HF, group fed high-fat diet; ST, treatment group fed high-fat diet and trained with swimming from week 4; SP, prevention group fed highfat diet and trained with swimming from the start of the experiment. FAT, fatty acid translocase; FABP, fatty acid-binding protein; CPT1, carnitine palmitoyltransferase 1; PPAR, peroxisome proliferator-activated receptor.

with the Con group. Following swinmming, the expression of these 4 factors was upregulated in the ST and SP groups compared with the HF group.

Swimming upregulates energy metabolism-related gene expression in skeletal muscle. In order to elucidate the effects of a high-fat diet, as well as swimming on the expression of energy metabolism-related genes, the AMPK $\alpha 2$, PGC1 $\alpha$, PGC1 $\beta$ and MFN2 levels were detected by quantitative RT-PCR and western blot analysis in the skeletal muscle of the rats. The results revealed that the expression of these 4 factors was downregulated in the skeletal muscle of the HF group rats compared with the Con group rats; following swimming, in the ST and SP groups, the expression of these 4 factors was markedly upregulated compared with the HF group (Fig. 5).
A
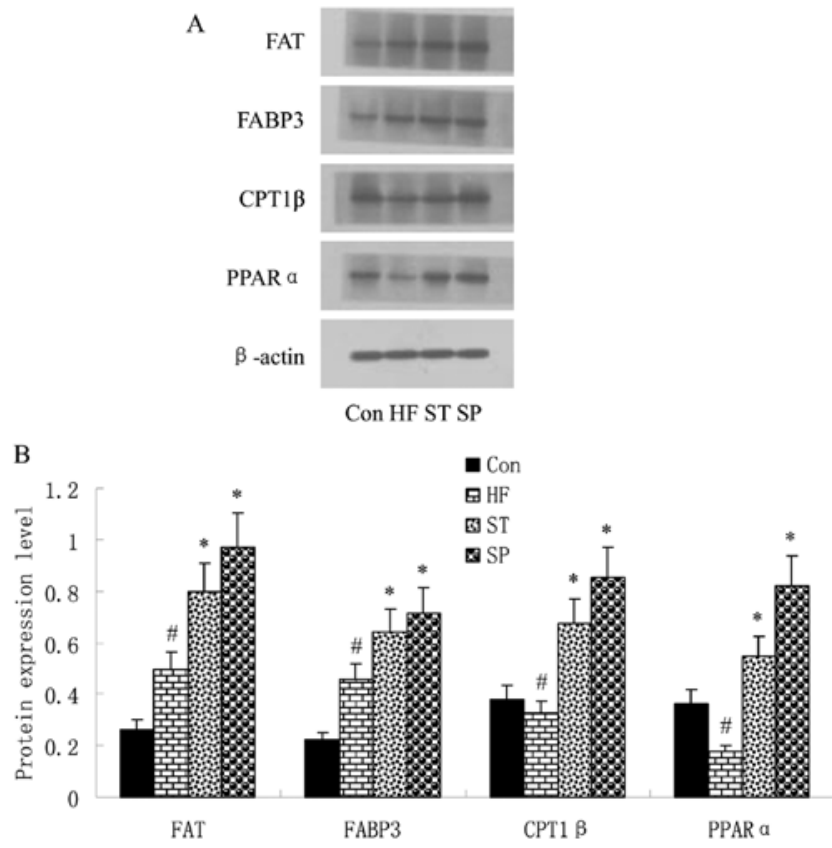

$\mathrm{C}$

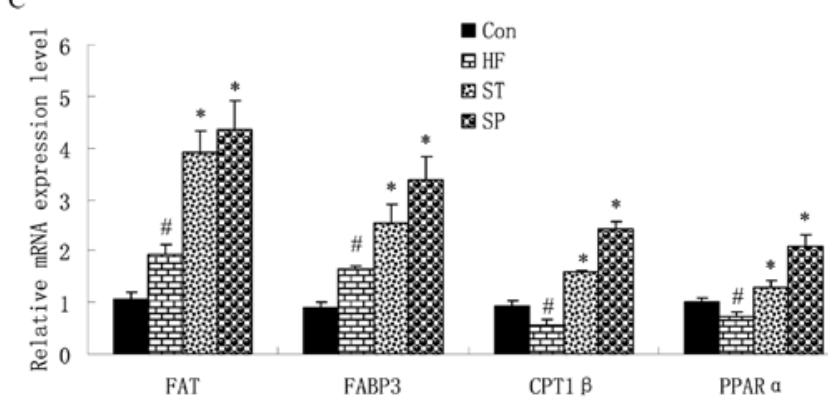

Figure 4. Swimming regulates fat metabolism-related gene expression in skeletal muscle. Rats were fed a control or high-fat diet for 8 weeks, and some were trained with swimming for 4 or 8 weeks, and were then sacrificed. Skeletal muscle of rats was taken immediately and subjected to (A) western blot analysis and (C) quantitative RT-PCR to determine the mRNA and protein expression levels of fat metabolism-related genes. The band density of western blots representing the target gene protein expression level is shown in (B). Experiments were repeated 3 times $(\mathrm{n}=3)$. ${ }^{~} \mathrm{P}<0.05$ vs. Con group; ${ }^{*} \mathrm{P}<0.05$ vs. HF group. Con, control group; HF, group fed high-fat diet; ST, treatment group fed high-fat diet and trained with swimming from week 4; SP, prevention group fed high-fat diet and trained with swimming from the start of the experiment. FAT, fatty acid translocase; FABP, fatty acid-binding protein; CPT1, carnitine palmitoyltransferase 1; PPAR, peroxisome proliferatoractivated receptor.

Swimming improves insulin signaling in skeletal muscle. The role of insulin-stimulated glucose utilization relies on the insulin signaling pathway, as this determines the level of insulin resistance in skeletal muscle. Therefore, the expression levels of insulin pathway-related factors, INSR, IRS1, PI3K/p85, AKT2 and GLUT4 in the skeletal muscle of the rats were determined by quantitative RT-PCR and western blot analysis.

As shown in Fig. 6, the mRNA and protein expression levels of INSR, IRS1, PI3K/p85, AKT2 and GLUT4 were lower in the skeletal muscle of the HF rats compared with those in the Con group rats. The phosphorylation levels of INSR and IRS1 were also decreased in the skeletal muscle of HF rats compared with those in the Con group rats. Although there were no marked changes in the protein levels of PI3K/p85 and AKT2, their phosphorylation levels were significantly lower in 


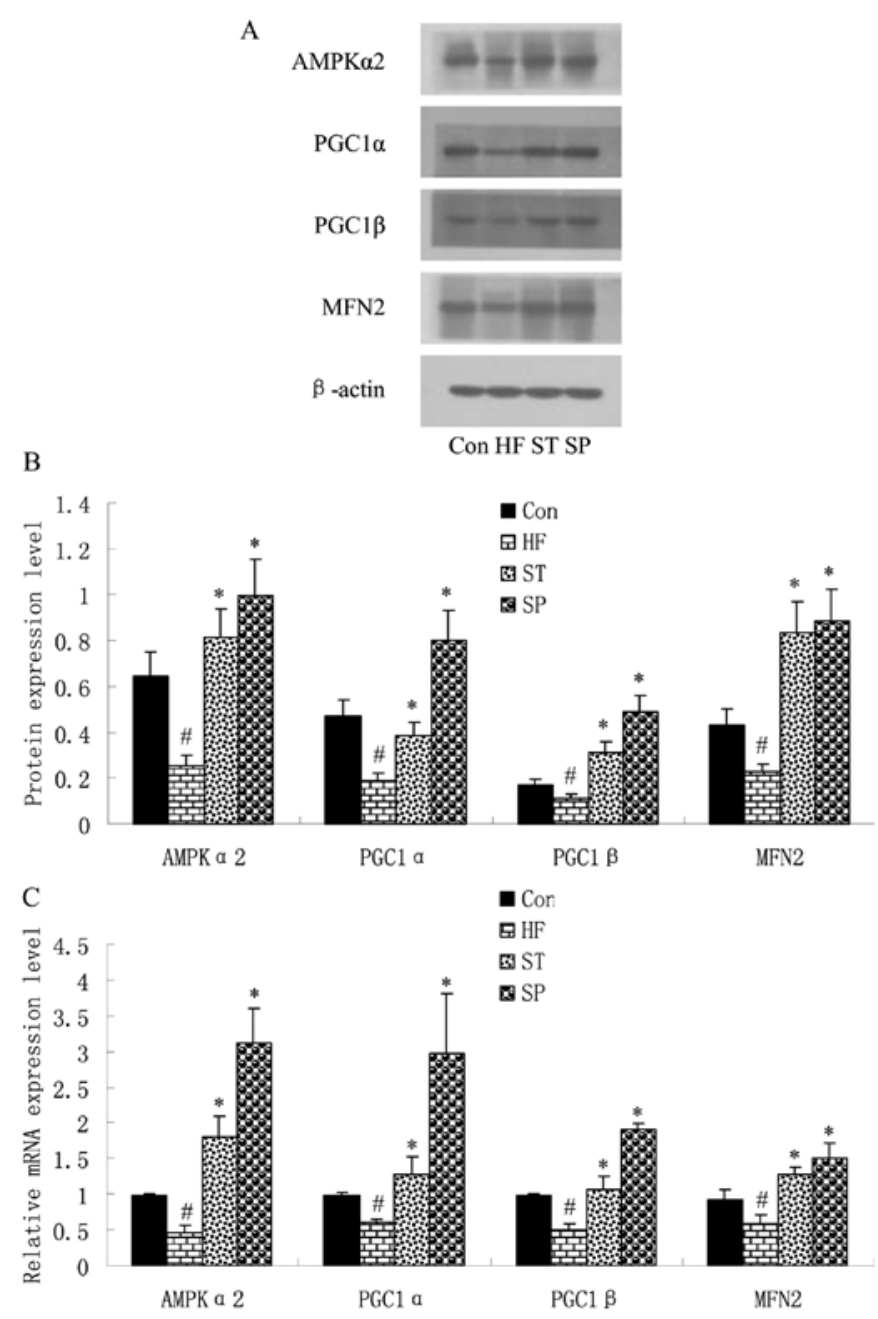

Figure 5. Swimming upregulates energy metabolism-related gene expression in skeletal muscle. Rats were fed a control or high-fat diet for 8 weeks, and some were trained with swimming for 4 or 8 weeks, and were then sacrificed. Skeletal muscle of rats was taken immediately and subjected to (A) western blot analysis and (C) quantitative RT-PCR to determine the mRNA and protein expression levels of energy metabolism-related genes. The band density of western blots representing the target gene protein expression level is shown in (B). Experiments were repeated 3 times $(n=3) .{ }^{*} \mathrm{P}<0.05$ vs. Con group; ${ }^{*} \mathrm{P}<0.05$ vs. HF group. Con, control group; HF, group fed high-fat diet; ST, treatment group fed high-fat diet and trained with swimming from week 4; SP, prevention group fed high-fat diet and trained with swimming from the start of the experiment. AMPK, AMP-activated protein kinase; PGC, peroxisome proliferator-activated receptor $\gamma$ co-activator; MFN, mitofusin.

HF rats compared with the Con group rats. Most importantly, the expression and phosphorylation levels of these genes in the skeletal muscle of the ST and SP group rats were much higher compared with those in the HF group rats.

\section{Discussion}

Studies have shown that a high-fat diet, particularly one rich in saturated fatty acids, causes fat to be easily deposited due to its interference with the oxidation and lipolysis process $(9,10)$. Studies employing magnetic resonance spectroscopy found that skeletal muscle lipid is associated with insulin resistance $(18,19)$. In healthy individuals without a family history of diabetes, insulin sensitivity experiments have shown that

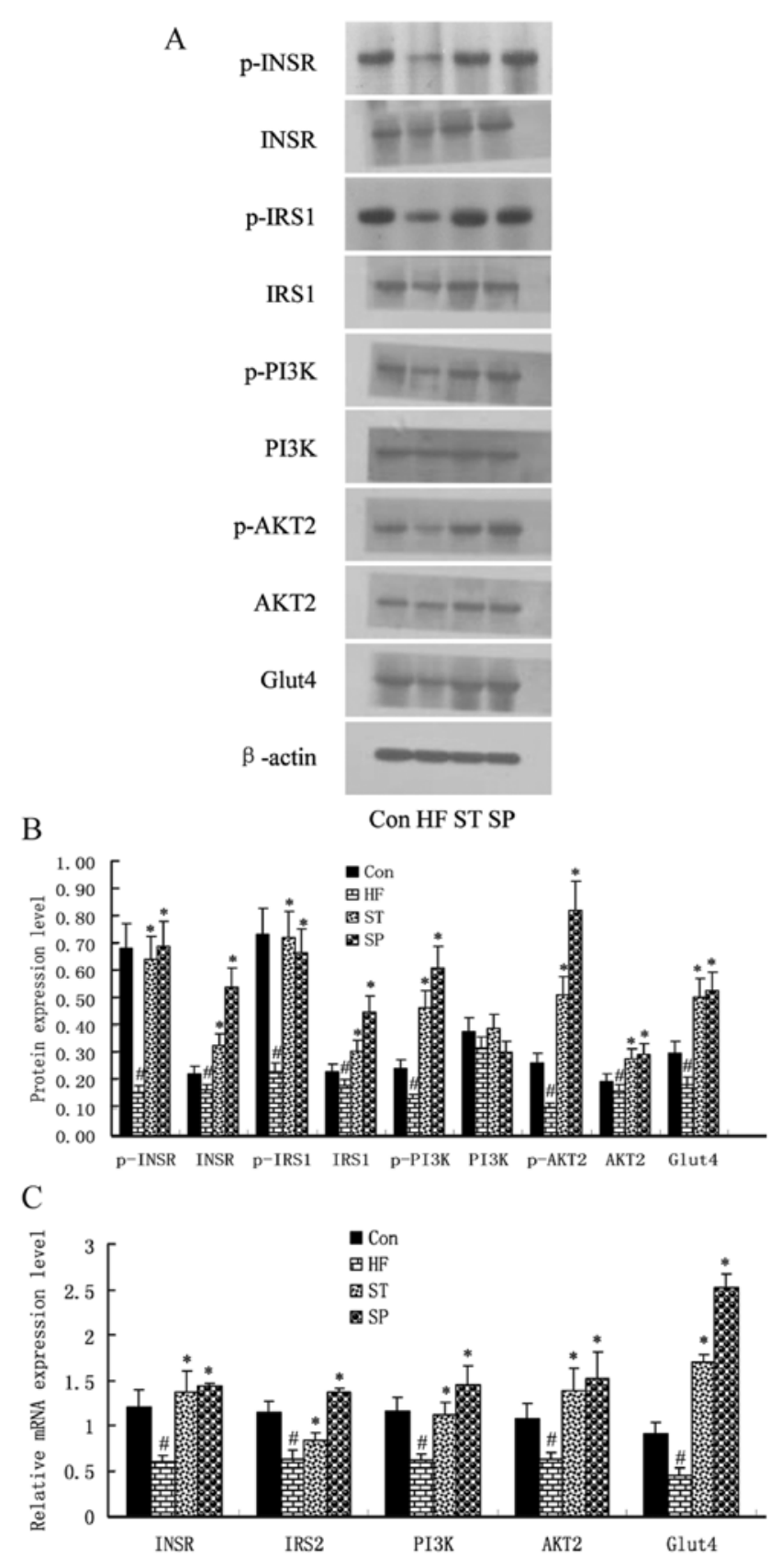

Figure 6. Swimming improves insulin signaling in skeletal muscle. Rats were fed a control or high-fat diet for 8 weeks, and some were trained with swimming for 4 or 8 weeks, and were then were. Skeletal muscle of rats was taken immediately and subjected to (A) western blot analysis and (C) quantitative RT-PCR to determine the mRNA and protein expression levels of insulin pathway-related genes. The band density of western blots representing the target gene protein expression level is shown in (B). Experiments were repeated 3 times $(n=3)$. ${ }^{~} \mathrm{P}<0.05$ vs. Con group; ${ }^{*} \mathrm{P}<0.05$ vs. HF group. Con group; ${ }^{\mathrm{P}}<0.05$ vs. HF group. Con, control group; HF, group fed high-fat diet; ST, treatment group fed high-fat diet and trained with swimming from week 4; SP, prevention group fed high-fat diet and trained with swimming from the start of the experiment. INSR, insulin receptor; IRS1, insulin receptor substrate 1; PI3K, phosphatidylinositol-3-kinas; GLUT4, glucose transporter 4.

insulin-stimulated glucose uptake is decreased in individuals with a higher skeletal muscle lipid content (20). Fat deposition may damage the cell membrane function, resulting in a lipid metabolism disorder, leading to insulin resistance through the inhibition of glucose transport and oxidation $(3,4)$. Insulin 
resistance, defined as the diminished ability of insulin-sensitive tissues, including liver, muscle and fat to respond to insulin, plays a central role in the development of type 2 diabetes mellitus (T2DM) and other metabolic diseases, such as obesity and metabolic syndrome (2). Insulin resistance is the primary predisposing factor for metabolic syndrome; it therefore becomes imperative to take effective measures in order to regulate lipid metabolism and prevent insulin resistance.

Our study, using rats, found that a high-fat diet leads to weight gain, liver fat deposition, ultrastructural damage to cells and a significant increase in the FBG, FINS, TG and TC levels in blood, thus causing severe insulin resistance. Importantly, our results revealed that swimming significantly improved the effects induced by a high-fat diet, and that swimming, as a preventive measure, produced a more effective result. Another study found that a long-term negative energy balance generated by exercise training significantly reduced plasma insulin levels, whereas insulin sensitivity was improved (21). There are also reports that endurance training can improve highfat diet-induced insulin resistance by increasing the insulin receptor binding activities of the liver and muscle cells (22).

The lipid content in skeletal muscle and liver is determined by the dynamic equilibrium between the uptake and disposal of fatty acids (23). FAT binds long-chain fatty acids and may function in the transport and/or as a regulator of fatty acid transport (24). After entering into cells, fatty acids are converted into TGs or are channeled towards the $\beta$-oxidaton pathway. The entry of fatty acyl-CoA into the mitochondria is the rate-limiting step for mitochondrial fatty acid oxidation, but long-chain fatty acyl-CoA (LCACoA) cannot permeate the mitochondrial membrane directly. Acyl-CoA first reacts with carnitine by the catalysis of CPT-1, and then enters the mitochondrial matrix (25). FABP is essential for fatty acid transport and PPAR plays a key role in fatty acid metabolism in the liver and skeletal muscle (26-29). Thus, some abnormal steps involved in fatty acid metabolism may contribute to fat accumulation in skeletal muscle and the liver. Our study suggests that a high-fat diet leads to increased levels of FAT and FABP, and decreased levels of PPAR and CPT1; furthermore, swimming upregulates the expressoin of these factors, as it is helpful for lipid cleaning from tissues.

Skeletal muscle, the largest movement system, is an important organ for energy metabolism. Studies have shown that AMPK is a phylogenetically-conserved intracellular energy sensor (30,31). AMPK can also regulate malonyl CoA levels by inhibitting acetyl-coenzyme A carboxylase (ACC) and activating malonyl CoA decarboxylase (MCD) in muscle, and has been implicated in the regulation of energy metabolism (32). MFN2, a mitochondrial fusion protein, is involved in mitochondrial morphology and metabolism regulation (33-35). The transcription factors, PGC- $1 \alpha$ and $\beta$, are considered master metabolic regulators and promoters of mitochondrial biogenesis $(36,37)$. Specifically, the activation of AMPK signaling cascades are well-characterized upstream modulators of PGC-1 $\alpha$ gene expression in skeletal muscle (38-40). Therefore, the abnormal expression or activity of these signaling molecules may contribute to fat accumulation and insulin resistance in skeletal muscle. The regulation of the AMPK pathway may reduce ectopic lipid accumulation, improving insulin resistance, and thereby mediating beneficial effects in various diseases associated with insulin resistance. The present study demonstrates that a high-fat diet leads to a decrease in AMPK, PGC1 $\alpha$, PGC1 $\beta$ and MFN2 expression, while swimming upregulates the expression of these genes in skeletal muscle. The results suggest that swimming improves lipid deposition and insulin resistance by regulating energy metabolism.

Skeletal muscle tissue is the most important organ where insulin-mediated glucose uptake occurs, as $>90 \%$ of insulin-mediated glucose uptake in the body is by skeletal muscles. Therefore, the ability of skeletal muscle glucose utilization determines the level of insulin resistance. The utilization of glucose by skeletal muscle stimulated with insulin occurs through a series of signaling processes of the INSR/ IRS/PI3K/AKT/GLUT4 pathway $(41,42)$. Finally, GLUT4 translocates to the cell membrane and accelerates glucose uptake into the cell (43). Abnormality in any steps of the insulin signal transduction pathway may eventually impede glucose transportation in skeletal muscle, thereby causing insulin resistance. This study demonstrates that a high-fat diet inhibits the expression of INSR, IRS1, PI3K/p85, AKT2 and GLUT4, particularly the phosphorylation of PI3K/p85 and AKT2. Moreover, swimming restores the levels of these factors in skeletal muscle, as it improves insulin resistance by strengthening the insulin signaling pathway.

In conclusion, swimming improve high-fat-induced insulin resistance by reducing liver and muscle lipid accumulation and by regulating energy metabolism and the insulin signaling pathway in skeletal muscle.

\section{Acknowledgements}

The authors would like to thank Professor Guangyao Song (Department of Endocrinology, General Hospital of Hebei, Shijiazhuang, Hebei, P.R. China) for providing his advice. The present study was supported by grants from the National Natural Science Foundation of China (30971391, 81170742) and the Hebei Natural Science Foundation of China (C2010001638, C2011307008).

\section{References}

1. Karpe F, Dickmann JR and Frayn KN: Fatty acids, obesity, and insulin resistance: time for a reevaluation. Diabetes 60: 2441-2449, 2011

2. Wang ZL, Xia B, Shrestha U, et al: Correlation between adiponectin polymorphisms and non-alcoholic fatty liver disease with or without metabolic syndrome in Chinese population. J Endocrinol Invest 31: 1086-1091, 2008.

3. Chow L, From A and Seaquist E: Skeletal muscle insulin resistance: the interplay of local lipid excess and mitochondrial dysfunction. Metabolism 59: 70-85, 2010.

4. Martins AR, Nachbar RT, Gorjao R, et al: Mechanisms underlying skeletal muscle insulin resistance induced by fatty acids: importance of the mitochondrial function. Lipids Health Dis 11: 30, 2012.

5. Song GY, Ren LP, Chen SC, et al: Similar changes in muscle lipid metabolism are induced by chronic high-fructose feeding and high-fat feeding in C57BL/J6 mice. Clin Exp Pharmacol Physiol 39: 1011-1018, 2012.

6. Iellamo F, Caminiti G, Sposato B, et al: Effect of high-intensity interval training versus moderate continuous training on $24-\mathrm{h}$ blood pressure profile and insulin resistance in patients with chronic heart failure. Intern Emerg Med: Jul 16, 2013 (Epub ahead of print).

7. Chowdhury KK, Legare DJ and Lautt WW: Interaction of antioxidants and exercise on insulin sensitivity in healthy and prediabetic rats. Can J Physiol Pharmacol 91: 570-577, 2013. 
8. Buettner R, Parhofer KG, Woenckhaus M, et al: Defining high-fat-diet rat models: metabolic and molecular effects of different fat types. J Mol Endocrinol 36: 485-501, 2006.

9. Bray GA, Lovejoy JC, Smith SR, et al: The influence of different fats and fatty acids on obesity, insulin resistance and inflammation. J Nutr 132: 2488-2491, 2002.

10. McGarry JD: Banting lecture 2001: dysregulation of fatty acid metabolism in the etiology of type 2 diabetes. Diabetes 51: 7-18, 2002.

11. Gan KX, Wang C, Chen JH, Zhu CJ and Song GY: Mitofusin-2 ameliorates high-fat diet-induced insulin resistance in liver of rats. World J Gastroenterol 19: 1572-1581, 2013.

12. Song GY, Gao Y, Wang C, et al: Rosiglitazone reduces fatty acid translocase and increases AMPK in skeletal muscle in aged rats: a possible mechanism to prevent high-fat-induced insulin resistance. Chin Med J (Engl) 123: 2384-2391, 2010.

13. Kong D, Song G, Wang C, et al: Overexpression of mitofusin 2 improves translocation of glucose transporter 4 in skeletal muscle of highfat dietfed rats through AMP activated protein kinase signaling. Mol Med Rep 8: 205-210, 2013.

14. Shulman GI: Cellular mechanisms of insulin resistance. J Clin Invest 106: 171-176, 2000.

15. Liu L, Zhang Y, Chen N, Shi X, Tsang B and Yu YH: Upregulation of myocellular DGAT1 augments triglyceride synthesis in skeletal muscle and protects against fat-induced insulin resistance. J Clin Invest 117: 1679-1689, 2007.

16. Schenk S and Horowitz JF: Acute exercise increases triglyceride synthesis in skeletal muscle and prevents fatty acid-induced insulin resistance. J Clin Invest 117: 1690-1698, 2007.

17. Wang C, Han M, Zhao XM and Wen JK: Kruppel-like factor 4 is required for the expression of vascular smooth muscle cell differentiation marker genes induced by all-trans retinoic acid J Biochem 144: 313-321, 2008.

18. Goodpaster BH, Krishnaswami S, Resnick H, et al: Association between regional adipose tissue distribution and both type 2 diabetes and impaired glucose tolerance in elderly men and women. Diabetes Care 26: 372-379, 2003.

19. Perseghin G, Scifo P, De Cobelli F, et al: Intramyocellular triglyceride content is a determinant of in vivo insulin resistance in humans: a $1 \mathrm{H}-13 \mathrm{C}$ nuclear magnetic resonance spectroscopy assessment in offspring of type 2 diabetic parents. Diabetes 48 : 1600-1606, 1999.

20. Virkamaki A, Korsheninnikova E, Seppala-Lindroos A, et al: Intramyocellular lipid is associated with resistance to in vivo insulin actions on glucose uptake, antilipolysis, and early insulin signaling pathways in human skeletal muscle. Diabetes 50: 2337-2343, 2001

21. Oppert JM, Nadeau A, Tremblay A, Despres JP, Theriault G and Bouchard C: Negative energy balance with exercise in identical twins: plasma glucose and insulin responses. Am J Physiol 272: E248-E254, 1997.

22. Corcoran MP, Lamon-Fava S and Fielding RA: Skeletal muscle lipid deposition and insulin resistance: effect of dietary fatty acids and exercise. Am J Clin Nutr 85: 662-677, 2007.

23. Samuel VT, Petersen KF and Shulman GI: Lipid-induced insulin resistance: unravelling the mechanism. Lancet 375: 2267-2277, 2010.

24. Campbell SE, Tandon NN, Woldegiorgis G, Luiken JJ, Glatz JF and Bonen A: A novel function for fatty acid translocase (FAT)/ CD36: involvement in long chain fatty acid transfer into the mitochondria. J Biol Chem 279: 36235-36241, 2004.

25. Sebastian D, Herrero L, Serra D, Asins G and Hegardt FG: CPT I overexpression protects L6E9 muscle cells from fatty acid-induced insulin resistance. Am J Physiol Endocrinol Metab 292: E677-E686, 2007.
26. Smathers RL and Petersen DR: The human fatty acid-binding protein family: evolutionary divergences and functions. Hum Genomics 5: 170-191, 2011.

27. Storch J and Thumser AE: Tissue-specific functions in the fatty acid-binding protein family. J Biol Chem 285: 32679-32683, 2010.

28. Kusudo T, Kontani Y, Kataoka N, Ando F, Shimokata H and Yamashita H: Fatty acid-binding protein 3 stimulates glucose uptake by facilitating AS160 phosphorylation in mouse muscle cells. Genes Cells 16: 681-691, 2011.

29. Ruderman NB, Carling D, Prentki M and Cacicedo JM: AMPK, insulin resistance, and the metabolic syndrome. J Clin Invest 123: 2764-2772, 2013

30. Cao S, Li B, Yi X, et al: Effects of exercise on AMPK signaling and downstream components to PI $3 \mathrm{~K}$ in rat with type 2 diabetes. PLoS One 7: e51709, 2012.

31. Velkov T: Interactions between human liver fatty acid binding protein and peroxisome proliferator activated receptor selective drugs. PPAR Res 2013: 938401, 2013.

32. Kramer DK, Al-Khalili L, Guigas B, Leng Y, Garcia-Roves PM and Krook A: Role of AMP kinase and PPARdelta in the regulation of lipid and glucose metabolism in human skeletal muscle. J Biol Chem 282: 19313-19320, 2007.

33. Liesa M, Palacin M and Zorzano A: Mitochondrial dynamics in mammalian health and disease. Physiol Rev 89: 799-845, 2009.

34. Sebastian D, Hernandez-Alvarez MI, Segales J, et al: Mitofusin 2 (Mfn2) links mitochondrial and endoplasmic reticulum function with insulin signaling and is essential for normal glucose homeostasis. Proc Natl Acad Sci USA 109: 5523-5528, 2012.

35. Zorzano A, Liesa M and Palacin M: Mitochondrial dynamics as a bridge between mitochondrial dysfunction and insulin resistance. Arch Physiol Biochem 115: 1-12, 2009.

36. Hernandez-Alvarez MI, Thabit H, Burns N, et al: Subjects with early-onset type 2 diabetes show defective activation of the skeletal muscle PGC-1\{alpha\}/Mitofusin-2 regulatory pathway in response to physical activity. Diabetes Care 33: 645-651, 2010.

37. Zorzano A, Hernandez-Alvarez MI, Palacin M and Mingrone G: Alterations in the mitochondrial regulatory pathways constituted by the nuclear co-factors PGC-1alpha or PGC-1beta and mitofusin 2 in skeletal muscle in type 2 diabetes. Biochim Biophys Acta 1797: 1028-1033, 2010.

38. Akimoto T, Pohnert SC, Li P, et al: Exercise stimulates Pgc-1alpha transcription in skeletal muscle through activation of the p38 MAPK pathway. J Biol Chem 280: 19587-19593, 2005.

39. Wright DC, Geiger PC, Han DH, Jones TE and Holloszy JO: Calcium induces increases in peroxisome proliferator-activated receptor gamma coactivator-1alpha and mitochondrial biogenesis by a pathway leading to $\mathrm{p} 38$ mitogen-activated protein kinase activation. J Biol Chem 282: 18793-18799, 2007.

40. Egan B, Carson BP, Garcia-Roves PM, et al: Exercise intensity-dependent regulation of peroxisome proliferator-activated receptor coactivator- 1 mRNA abundance is associated with differential activation of upstream signalling kinases in human skeletal muscle. J Physiol 588: 1779-1790, 2010.

41. Saltiel AR and Kahn CR: Insulin signalling and the regulation of glucose and lipid metabolism. Nature 414: 799-806, 2001.

42. Previs SF, Withers DJ, Ren JM, White MF and Shulman GI: Contrasting effects of IRS-1 versus IRS-2 gene disruption on carbohydrate and lipid metabolism in vivo. J Biol Chem 275: 38990-38994, 2000

43. Osorio-Fuentealba C, Contreras-Ferrat AE, Altamirano F, et al: Electrical stimuli release ATP to increase GLUT4 translocation and glucose uptake via PI3Kgamma-Akt-AS160 in skeletal muscle cells. Diabetes 62: 1519-1526, 2013. 